\title{
Adolescent Girls' Self-Concept and Its Related Factors Based on Roy Adaptation Model
}

\section{ART ICLE INFO}

\section{Article Type}

Descriptive Study

\section{Authors}

Basiri Moghadam M. ${ }^{1} M S c$, Khosravan Sh. ${ }^{2} P h D$

Sadeghmoghadam L. ${ }^{2} P h D$ Ebrahimi Senoo N.* MSC

How to cite this article
Basiri Moghadam M, Khosravan
Sh, Sadeghmoghadam L, Ebrahimi
Senoo N. Adolescent Girls' Self-
Concept and Its Related Factors
Based on Roy Adaptation Model.
Horizon of Medical Sciences.
2017;23(1):77-83.

*"Student Research Committee" and "Pediatrics Nursing Department, Nursing \& Midwifery Faculty", Gonabad University of Medical Sciences, Gonabad, Iran

${ }^{1 “}$ Health Promotion \& Social Development Research Center" and "Pediatrics Nursing Department, Nursing \& Midwifery Faculty", Gonabad University of Medical Sciences, Gonabad, Iran

${ }^{2 " S o c i a l ~ D e t e r m i n a t i o n ~ o f ~ H e a l t h ~}$ Research Center" and "Community \& Mental Health Nursing Department, Nursing \& Midwifery Faculty", Gonabad University of Medical Sciences, Gonabad, Iran

\section{Correspondence}

Address: Gonabad University of Medical Sciences, Near the Asian Road, Khorasan Razavi, Gonabad, Iran

Phone: +98 (51) 57225813

Fax: +98 (51) 57223813

ebrahimi.n67@gmail.com

\section{Article History}

Received: July 21, 2016

Accepted: October 13, 2016

ePublished: January 19, 2017

\section{A B S T R A C T}

Aims One of the most important factors of individual health in the adolescents is the selfconcept. As a nursing model, the Roy adaptation model mainly investigates the factor. The aim of the study was to investigate the self-concept and its related factors in the adolescent girls in Gonabad Township, based on the Roy adaptation model.

Instrument \& Methods In the descriptive cross-sectional study, 270 adolescent girls were studied in Gonabad Township, Iran, in 2015. The subjects were selected from three healthcare centers via stratified cluster random sampling method. Data was collected using a demographic questionnaire and a researcher-made inventory assessing the self-concept in the adolescent girls based on the Roy adaptation model. Data was analyzed by SPSS 22 software using descriptive statistics and Spearman's correlational and Chi-square tests. Findings The mean self-concept score of the adolescent girls was 281.10 \pm 19.06 .125 girls $(46.6 \%)$ and 143 girls $(53.4 \%)$ were good and moderate, respectively. Age and the constant self-concept $(\mathrm{p}=0.03 ; \mathrm{r}=-0.13)$, the economic status and the moral-spiritual self-concept $(\mathrm{p}=0.02 ; \mathrm{r}=0.15)$, as well as the constant self-concept $(\mathrm{p}=0.02 ; \mathrm{r}=0.11)$, father's educational level and the constant self-concept $(\mathrm{p}=0.04 ; \mathrm{r}=0.12)$, and father's occupation and the mental self-concept $(\mathrm{p}=0.04 ; \mathrm{r}=0.13$ ) were significantly correlated.

Conclusion The self-concept is moderate in more than a half of the adolescent girls in Gonabad Township. In addition, most of them have weak or moderate objective self.

Keywords Self Concept; Adolescent; Roy Adaptation Model

\section{I T A T I O N L INKS}

[1] Gender, age, and behavior differences in early ... [2] Health concerns and risk behaviors among university ... [3] Enhancing management of mothers during adolescence of ... [4] Evaluating puberty health program effect on ... [5] Health information seeking among Mbararan adolescents: results from the ... [6] The effect of educational group therapy plan on ... [7] Adolescents' perceptions of health ... [8] Relationship between motor proficiency and self-concept in girl ... [9] Comparison the self-concept and behavior in adolescents without criminal record with ... [10] Brief report: The association between non-suicidal self-injury, self-concept and ... [11] Self-concept in normal and criminal male ... [12] Selfconcept disturbances: Cognitive vulnerability for early ... [13] Prosocialself-schemas, self-awareness, and children's ... [14] Preliminary study of puberty education in ... [15] Health related quality of life in ... [16] Girls growing through adolescence have a higher risk of poor ... [17] The study of mental health status in high school female students in ... [18] Determinant factors of mental health based on ... [19] Study of mental health and its relationship with family welfare in pre-university students in ... [20] Mental health status of individuals fifteen years and older in ... [21] The role of self-concept mode of Roy's adaptation model on ... [22] The effect of care plan application based on Roy ... [23] The effect of nursing care plan based on "Roy's adaptation model" on physiological adaptation in ... [24] The influence of self-concept on adherence to ... [25] The effects of the roy's adaptation model on primigravida women's self-concept: A randomized ... [26] Correlates of body dissatisfaction among Taiwanese ... [27] Parent, peer, andmedia ... [28] Half of rural girls aged 8 to ... [29] Body dissatisfaction, engagement in ... [30] Investigating the relationship between ... [31] Demographic, behavioral, and ... [32] Body image satisfaction in adolescent girls and ... [33] Anger in adolescent communities: How angry are ... [34] Moral identity as moral ideal self: Links to ... [35] Interpersonal communication among ... [36] Longitudinal analysis of adolescent NSSI: The role of intrapersonal and ... [37] The longitudinal influence of self-efficacy, communication, and parenting on spontaneous adolescent ... [38] The relation between self-concept and ... [39] The correlation of family ... 


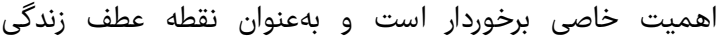

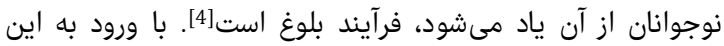

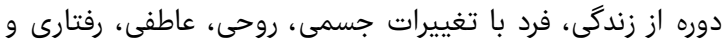

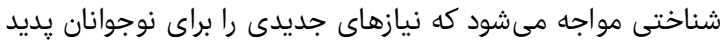

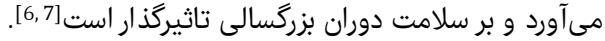

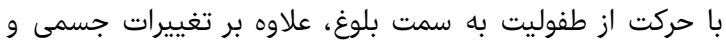

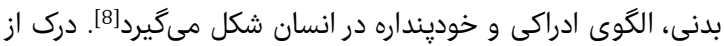

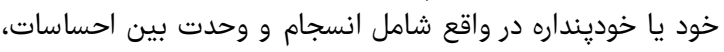

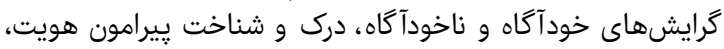

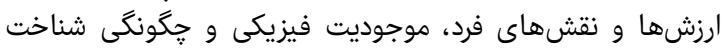

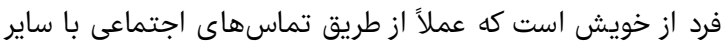

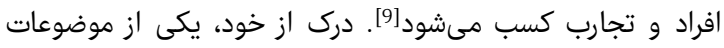

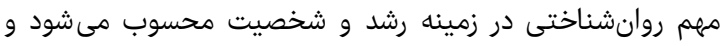

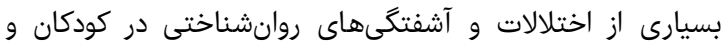

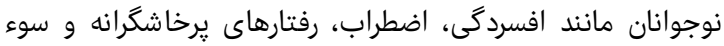

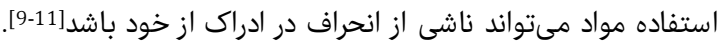

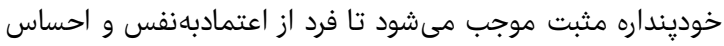

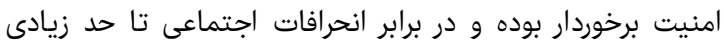

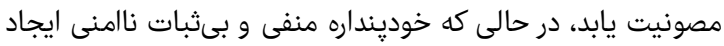

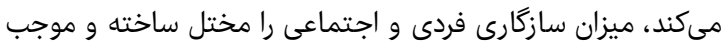

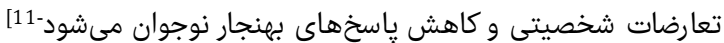

فرآيند بلوغ در دختران نوجوان از اهميت خاصى برخوردار است. در

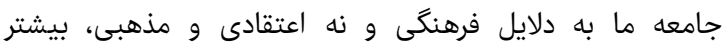

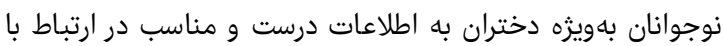

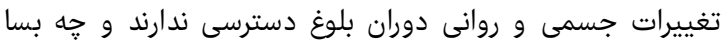

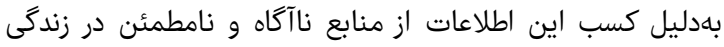

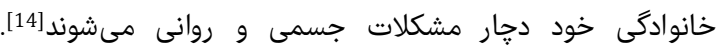

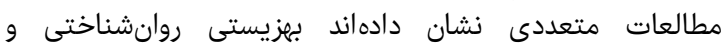

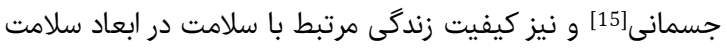

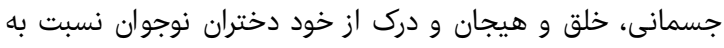

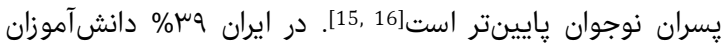

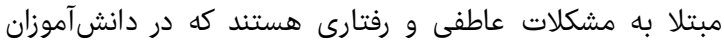

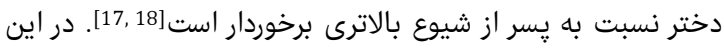

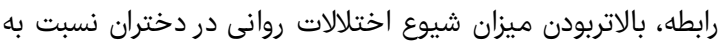

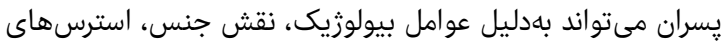

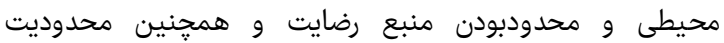

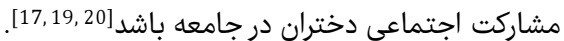

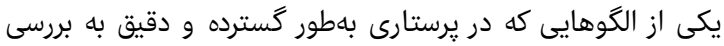

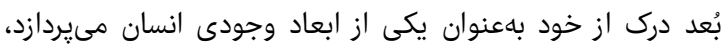

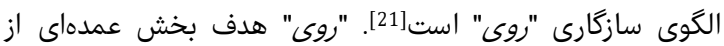

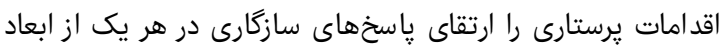

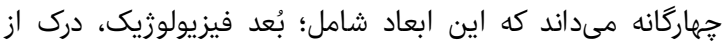

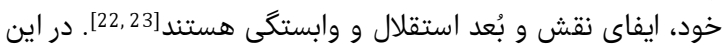

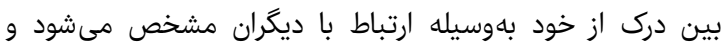

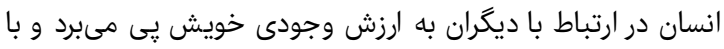

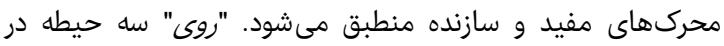

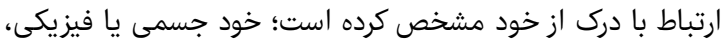

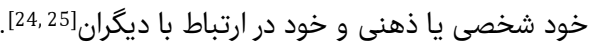

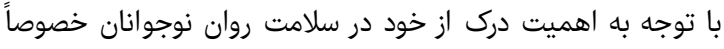

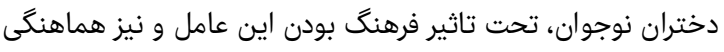

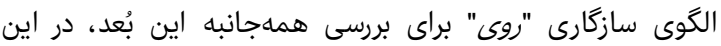

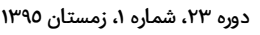

درك از خود و عوامل مرتبط با آن در دختران

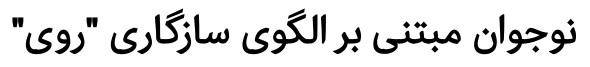

مهرى بصيرى مقدم MSc

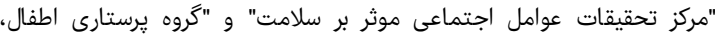

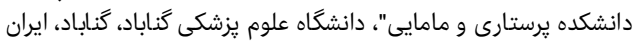

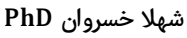

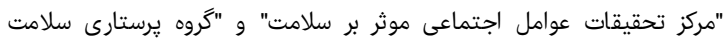

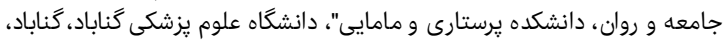

ايران

ليلا صادق مقدم PhD

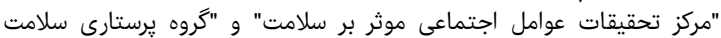

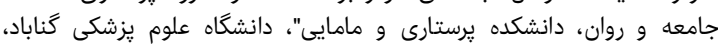

كناباد، ايران

MSc" نجمه ابراهيمى سنون ايران

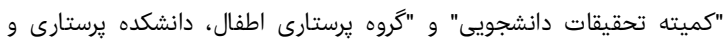
مامايى"، دانشكاه علوم يزشكى گناباد، تناباد، ايران

جكيده

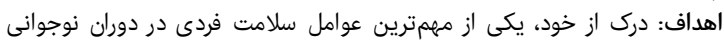

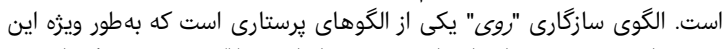

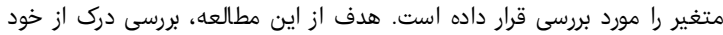

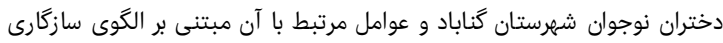

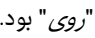

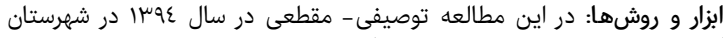
كناباد، • •

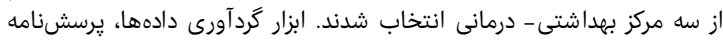

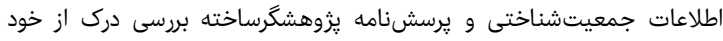

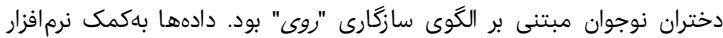
SPSS 22

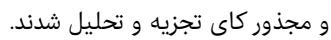

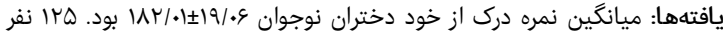

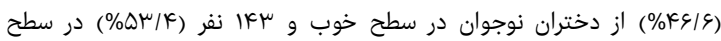

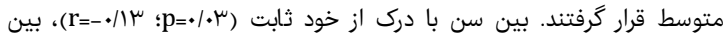

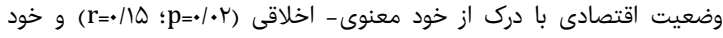

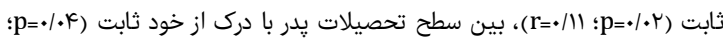

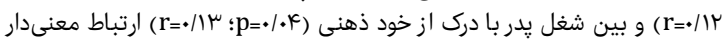

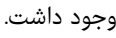

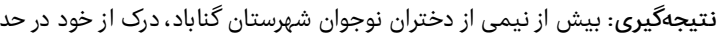

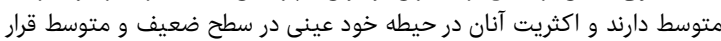

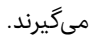
كليدوازمها: درك از خود، نوجوان، الكوى سازَارى "روى"

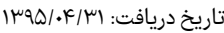

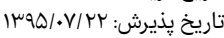
نويسنده مسئول: ebrahimi.n67@gmail.com

مقدمه - - مقدا

براساس تعريف سازمان جهانى بهداشت، دوران نوجوانى به سنين

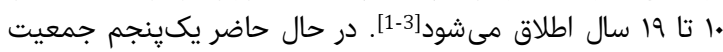

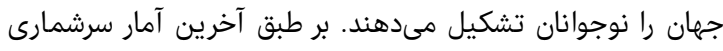

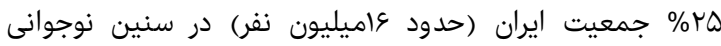

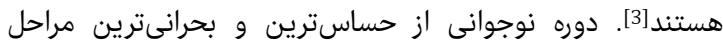

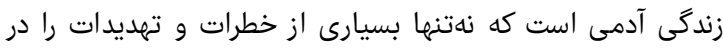

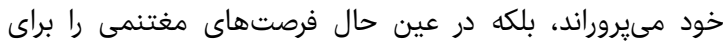

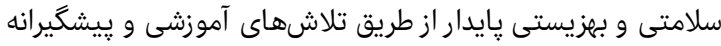

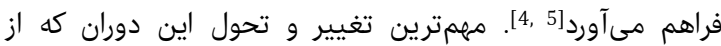

فصل نامه علمى - يُزوهشى افق دانش 


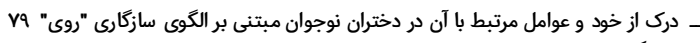

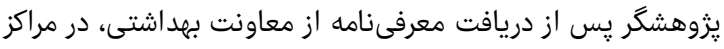

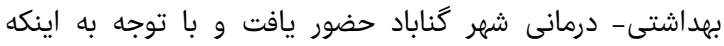

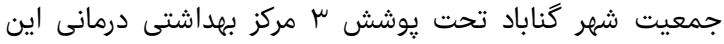

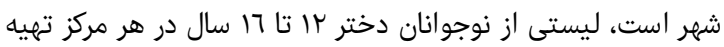

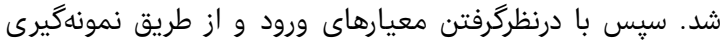

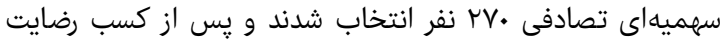

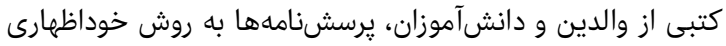
توسط شركت أكنندكان تكميل شدا داند

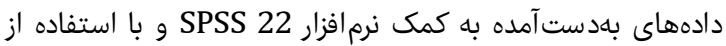

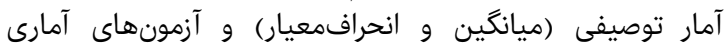

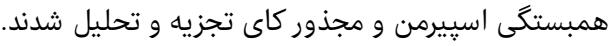

\section{يافتهها}

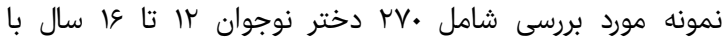

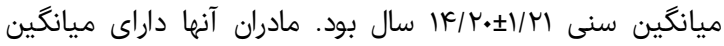

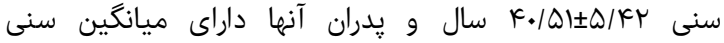


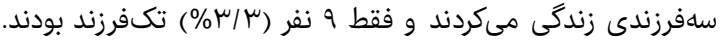

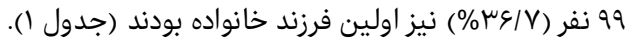

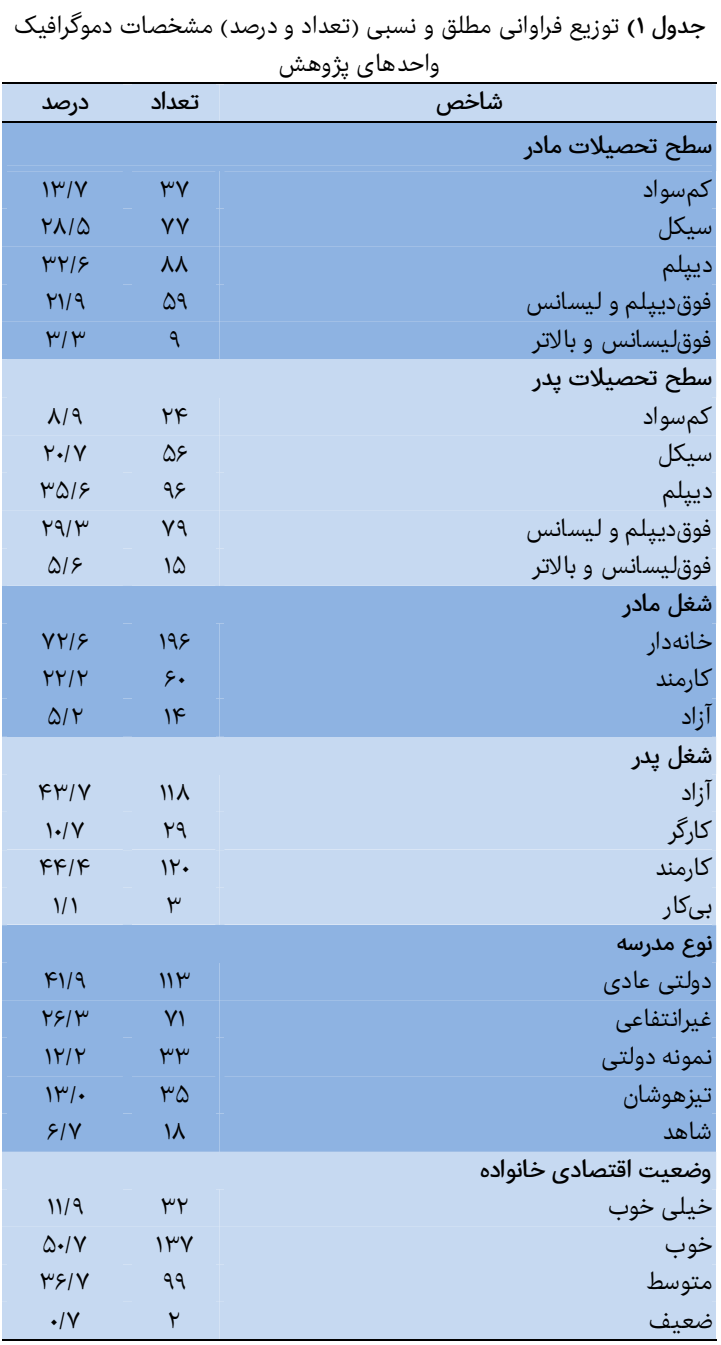

يثزوهش بر آن شديم تا درك از خود دردختران نوجوان شهر گناباد و

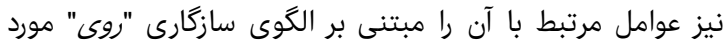

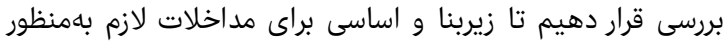

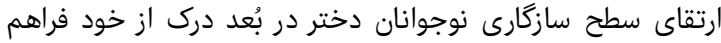

بنابراين هدف از اين مطالعه، بررسى درك از خود دختران نوجوان

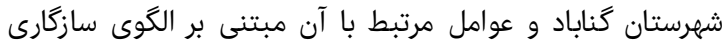

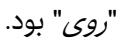

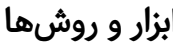

در اين مطالعه توصيفى- مقطعى در سال عوسا در شهرستان گناباد،

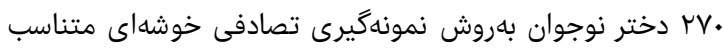

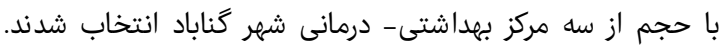

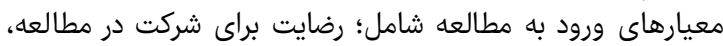

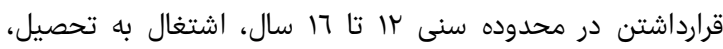

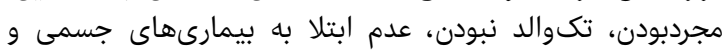

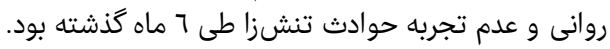

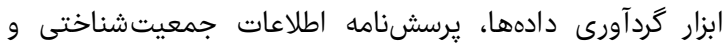

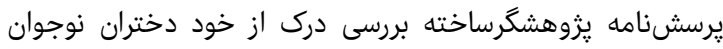

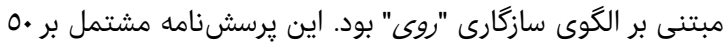

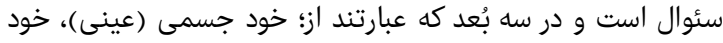

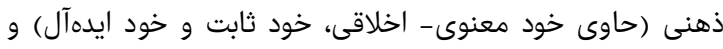

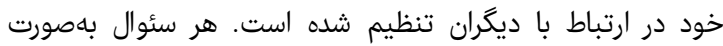

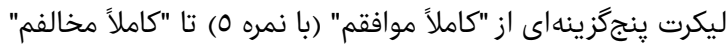

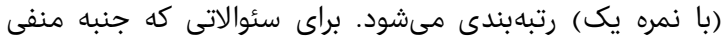

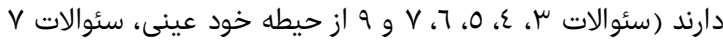

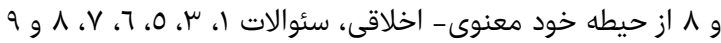

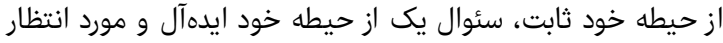

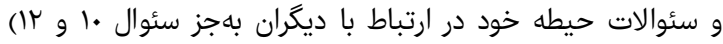

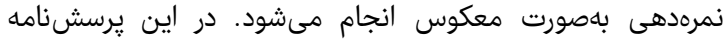

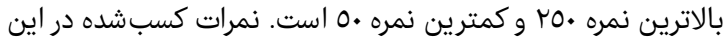

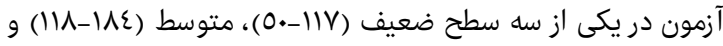

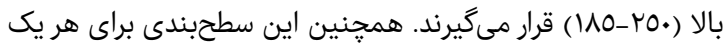

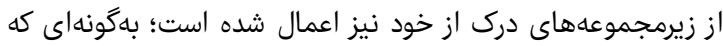

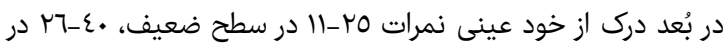

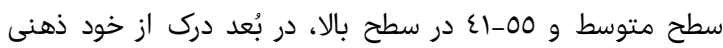
نمرات rV-7r در سطح ضعيف، و و

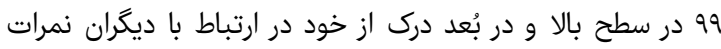
سطح

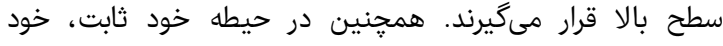

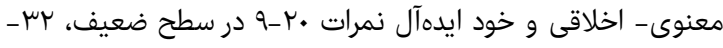

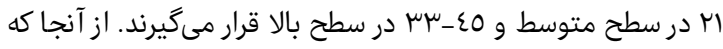

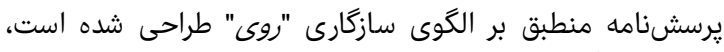

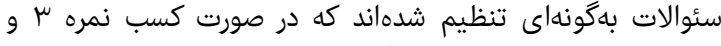

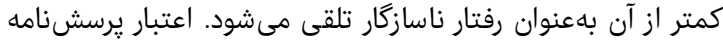

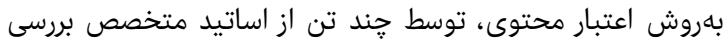

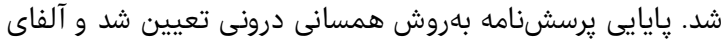

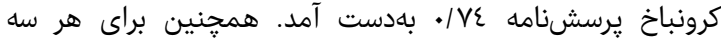

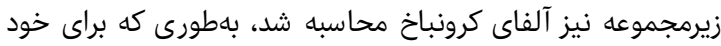

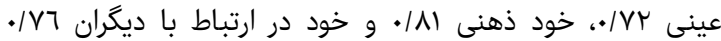

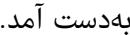


معنىدارى وجود داشت، بهطورى كه با افزايش وضعيت اقتصادى

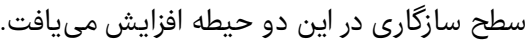

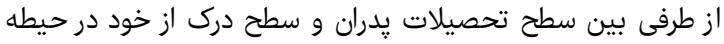

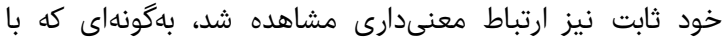

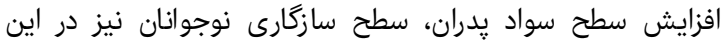

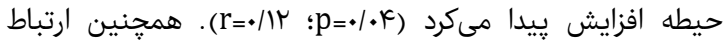

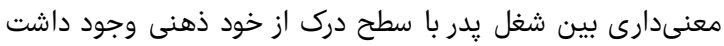

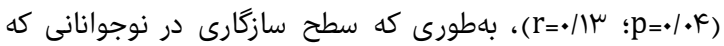

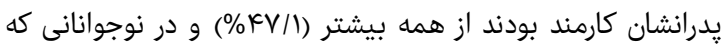

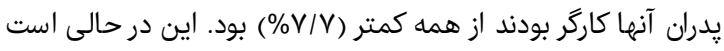

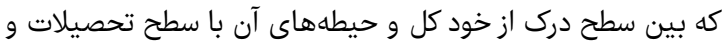

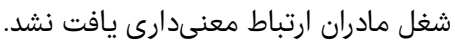

بحث

با توجه به نتايج مطالعه بيش از نيمى از دختران نوجوان به/R) به/R)

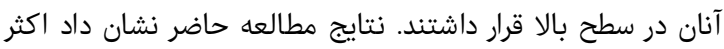

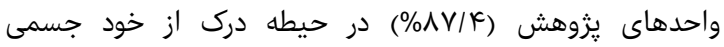

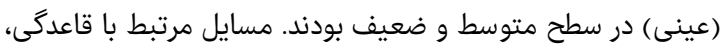

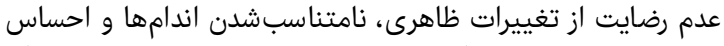

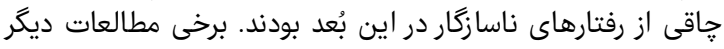

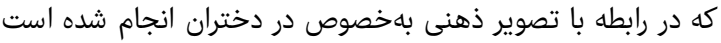

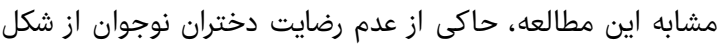

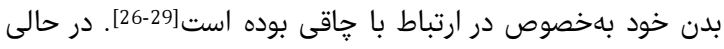

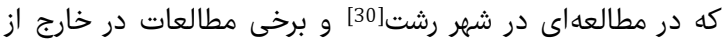

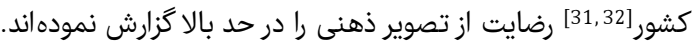
تصوير ذهنى از بدن خويش، تحت تاثير عوامل مختلفى از از جمله إنه

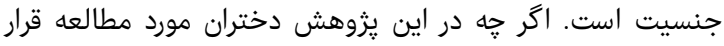

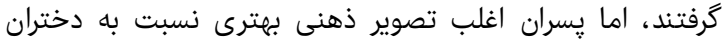

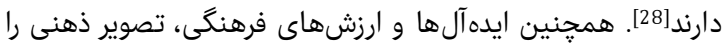

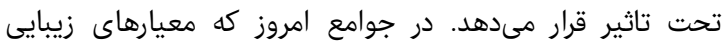

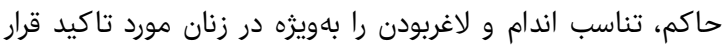

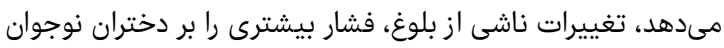

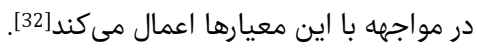

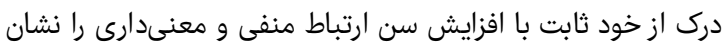

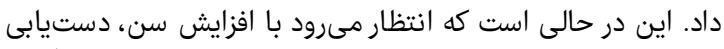

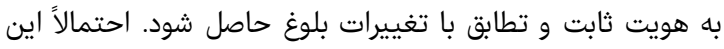

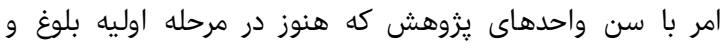

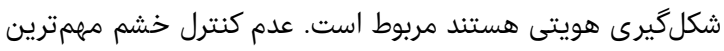

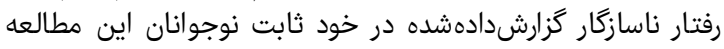

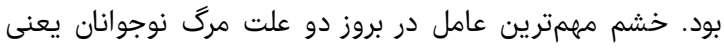

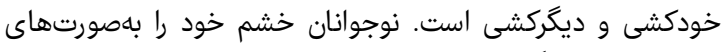

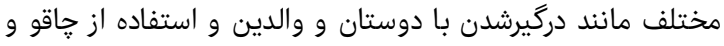

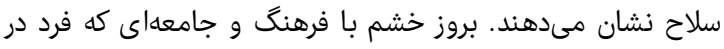

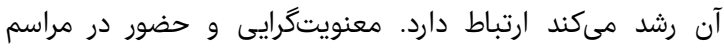

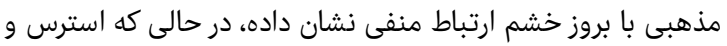

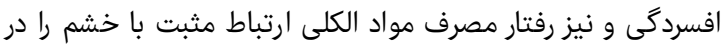

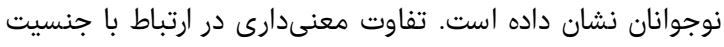

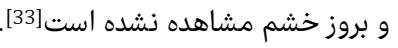

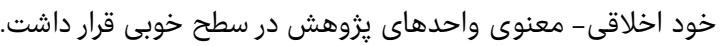

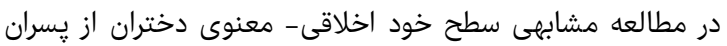

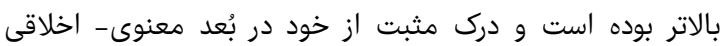

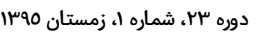

A مهدى بصيرى مقدم و همكاران

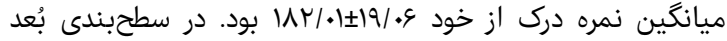

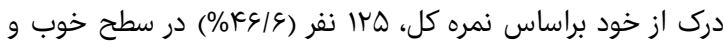
سط

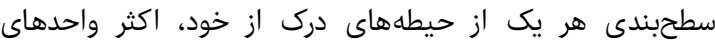

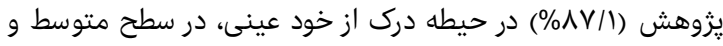

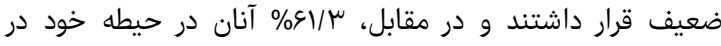

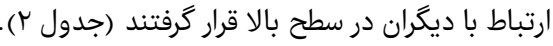

\begin{tabular}{|c|c|}
\hline \multicolumn{2}{|c|}{ يُزوهش (•rV نفر) } \\
\hline ميانكين آمارى & ابعاد درك از خود \\
\hline$\mu \kappa / \varepsilon r \pm \Delta / V$. & خود عينى \\
\hline $1.1 / \Delta Y \pm 11 / \mathrm{V}$. & خود ذهنى \\
\hline$\mu r / q \vee \pm \Delta / q \varphi$ & خود معنوى - اخلاقى \\
\hline$\mu \cdot / \vee \wedge \pm \Delta / \wedge q$ & خود ثابت \\
\hline$\mu \Delta / \vee \wedge \pm \varepsilon / I I$ & خود ايدهآل \\
\hline $\mathrm{K} \Delta / \wedge \Lambda \pm V / V q$ & خود در ارتباط با ديكران \\
\hline$|A r / \cdot| \pm 19 / \cdot 9$ & درك از خود (كل) \\
\hline
\end{tabular}

با توجه به نتايج كسبشده از يرسشنامه، شايعترين رفتارهاى

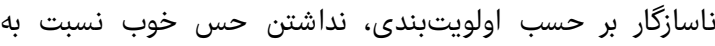

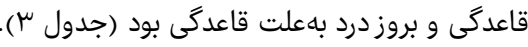

جدول ) اولويتبندى رفتارهاى ناسازگًار در حيطه هاى درك از خود

\begin{tabular}{|c|c|c|}
\hline تعداد & 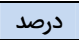 & رفتار ناسازگار \\
\hline & & 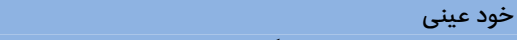 \\
\hline rrA & $\Delta \& / \Delta$ & حس خوبى نسبت به قاعدىى ندارم. \\
\hline rrs & $\Lambda \mu / \mathrm{V}$ & بروز قاعدگى باعث دردهاى مختلف در بدن من شده است. \\
\hline MIS & $\Lambda . \%$ & در دوران قاعدگى احساس كسالت و بىحوصلگى مىكنم. \\
\hline IVr & $8 \% \%$ & 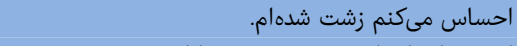 \\
\hline 190 & s//8 & از تغييرات ايجادشده در فرم بدنم ناراضى هستم. \\
\hline lQr & $\Delta \& / \mu$ & در مورد سلامتىام احساس نخرانى مىكنم. \\
\hline \multirow[t]{2}{*}{ IFs } & $\Delta F / /$ & احساس مىكنم جاق شدهام. \\
\hline & & خود ذهنى: زيرمولفه خود معنوى - اخلاقى \\
\hline $1 \wedge 1$ & $99 / 7$ & در دوران قاعدگى احساس ناياكى و دورى از خدا مىكنم. \\
\hline \multirow[t]{2}{*}{$1 \mu$. } & KN/Y & اعتقادم به مسايل مذهبى كمتر شده است. \\
\hline & & خود ذهنى: زيرمولفه خود ثابت \\
\hline IVY & $s \mu / \Lambda$ & فرد يرخاشكرى هستم. \\
\hline IKV & $\Delta F / Q$ & فردى مضطرب و يريشانم. \\
\hline \multirow[t]{2}{*}{111} & $k V / \Lambda$ & فردى كمرو و خجالتى هستم. \\
\hline & & خود ذهنى: زيرمولفه خود ايدهآل \\
\hline$|k|$ & $\Delta r / \mu$ & انتظار دارم ظاهرم بهتر از اين باشد. \\
\hline \multirow[t]{2}{*}{ Ir } & $\Delta 1 / 1$ & تمايلى ندارم استقلال كسب كنم. \\
\hline & & خود در ارتباط با ديكران \\
\hline$|Q|$ & $\Delta Q / 9$ & با اعضاى خانواده بهويزه مادرم مشاجره مىكنم. \\
\hline $1 \mu k$ & $k q / V$ & والدينم مرا خوب درك نمىكنند. \\
\hline irq & $k V / \Lambda$ & نمىتوانم احساساتم را بهراحتى با مادرم در ميان بكذارم. \\
\hline irr & $K \Delta / 1$ & والدينم بيش از حد مرا كنترل مىكنند. \\
\hline 111 & $k \mu / V$ & دوست ييداكردن برايم كار دشوارى است \\
\hline
\end{tabular}

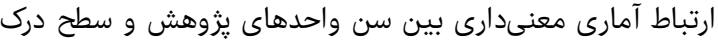

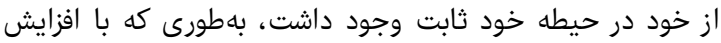

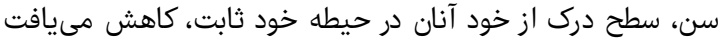

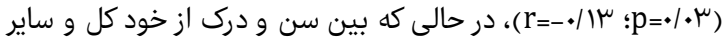

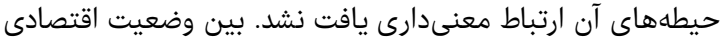

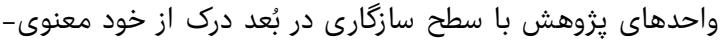

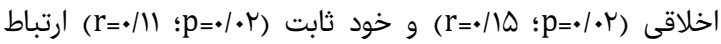

فصل نامه علمى - يزووهشى افق دانش 


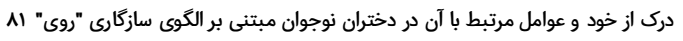

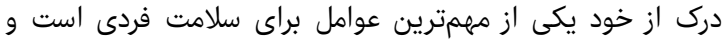

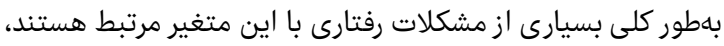

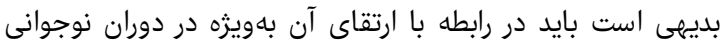

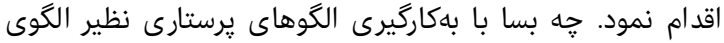

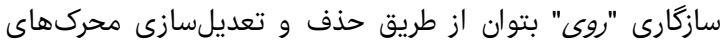

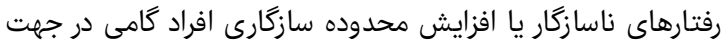

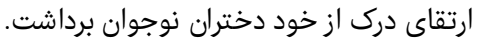

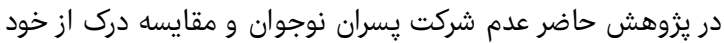

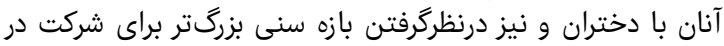

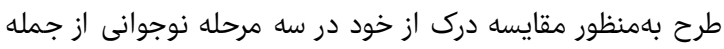

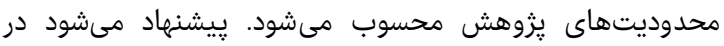

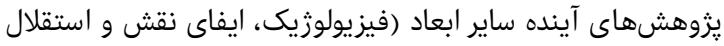

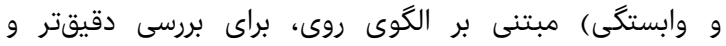

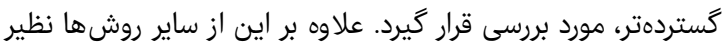

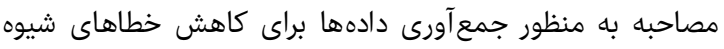
خوداظهارى در تكميل ابزارها استفاده شود.

\section{نتيجهكيرى}

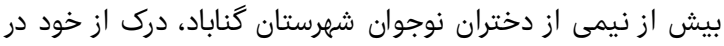

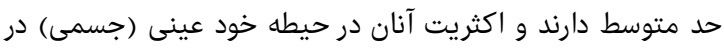

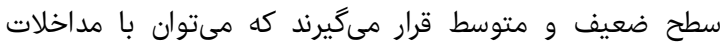

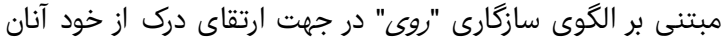

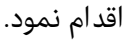

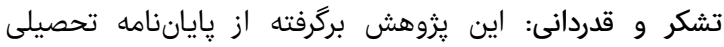

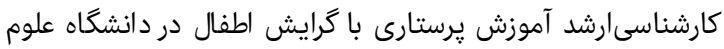
يزشكى گناباد با كد

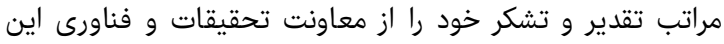

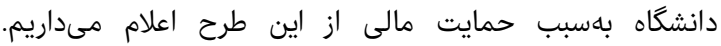

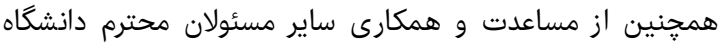

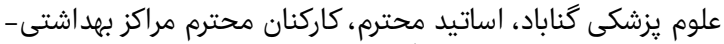

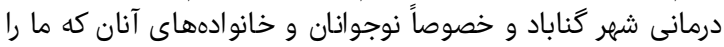

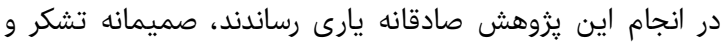
قدردانى مىنماييم. تاييديه اخلاقى: اين طرح با كد

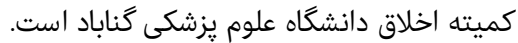

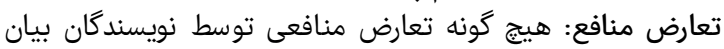

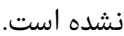

سهم نويسندكان: مهدى بصيرى مقدم (نويسنده اول) استاد راهنما

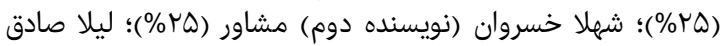

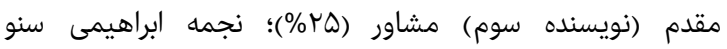

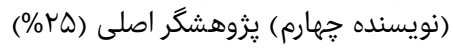

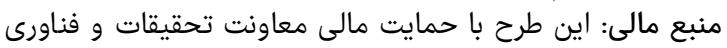
دانشكاه علوم يزشكى گَناباد انجام شده است.

منابع

1- Brown SL, Teufel JA, Birch DA, Kancherla V. Gender, age, and behavior differences in early adolescent worry. J Sch Health. 2006;76(8):430-7.

2- Hamdan-Mansour A. Health concerns and risk behaviors among university students in Jordan. Jordan Med J. 2007;41(2):80-90.

3- Ahangrzadeh Rezaei S, Khalilzadeh H. Enhancing management of mothers during adolescence of their

Horizon of Medical Sciences
بهاعنوان متغير يِيشبين رفتارهايى مانند كاهش احساس خشم و

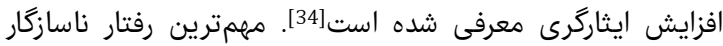

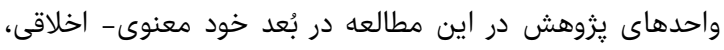

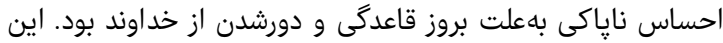

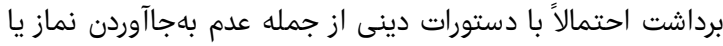

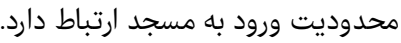

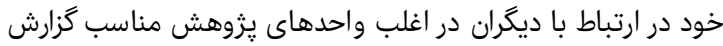

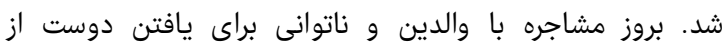

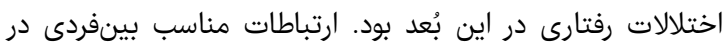

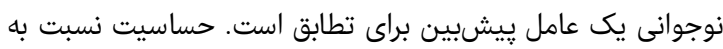

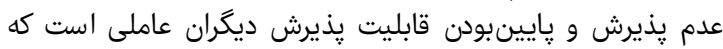

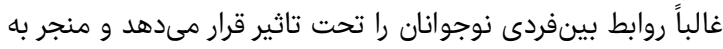

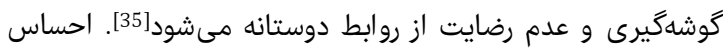

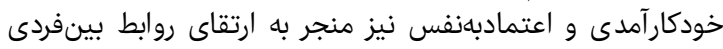

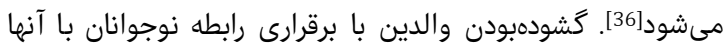

ارتباط دارد[37]. - مئ.

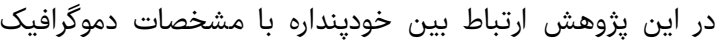
واحدهاى يزوهش بررسى شد. در مطالعه حاضر اكثريت نوجوانان (\% \%./V)

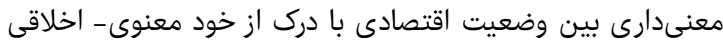

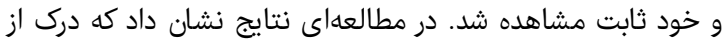

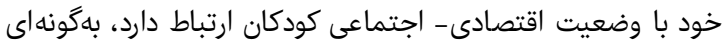

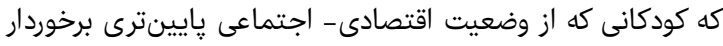

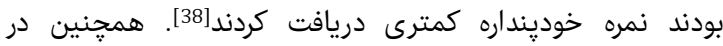

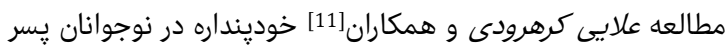

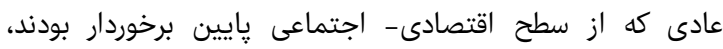

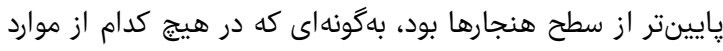

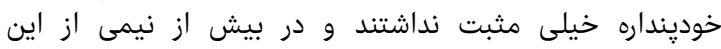

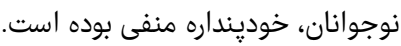

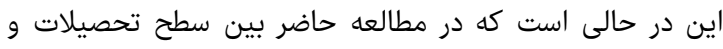

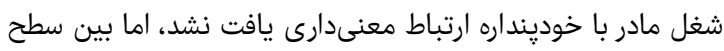

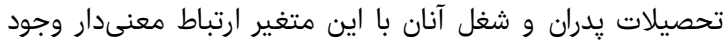

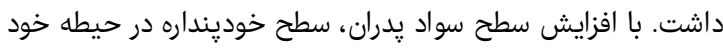

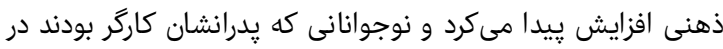

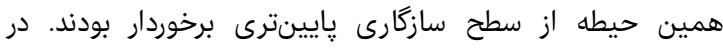

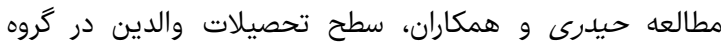

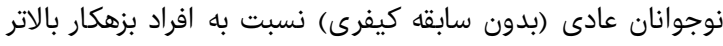

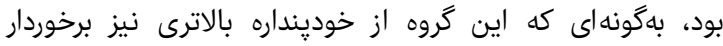

بودند [9].

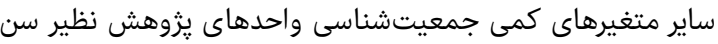

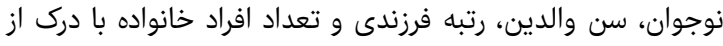

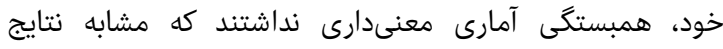

مطالعه حسينى و همكاران بود [39.

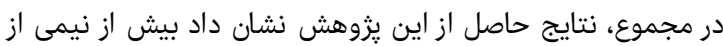

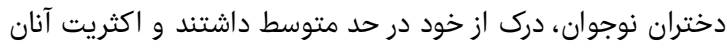

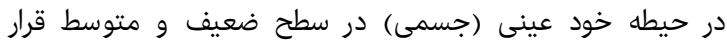

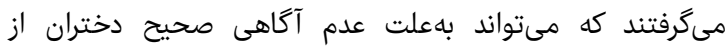

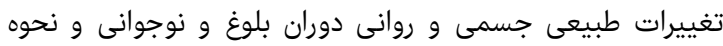

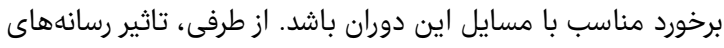

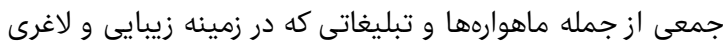

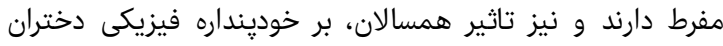

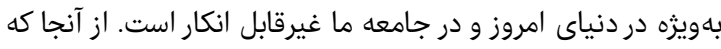


20- Noorbala AA, Bagheri Yazdi SA, Asadi Lari M, Vaez Mahdavi MR,. Mental health status of individuals fifteen years and older in Tehran-Iran. Iran J Psychiatry Clin Psychol. 2011;16(4):479-83. [Persian]

21- Ahrari Sh, Heydari A, Vaghee S. The role of selfconcept mode of Roy's adaptation model on adherence of diet regimen in heart failure patients. Horizon Med Sci. 2012;17(4):18-24. [Persian]

22- Maghsoodi E, Hemmati M, Sheikhi S, Khalkhali HR. The effect of care plan application based on Roy adaptation model on the self-esteem in elderly people in Urmia nursing homes. J Nurs Midwifery Urmia Univ Med Sci. 2015;12(10):926-34. [Persian]

23-Sadeghnezhad M, Vanaki Z, Memarian R. The effect of nursing care plan based on "Roy's adaptation model" on physiological adaptation in patients with type II diabetes. Daneshvar. 2011;18(92):25-34. [Persian]

24- Thomas CM. The influence of self-concept on adherence to recommended health regimens in adults with heart failure. J Cardiovasc Nurs. 2007;22(5):405-16. 25- Mohammadpour A, Najafi S, Tavakkolizadeh J, Mohammadzadeh F. The effects of the roy's adaptation model on primigravida women's self-concept: A randomized controlled trial. Asian J Pharm Res Health Care. 2016;8(Suppl 1):17-23.

26- Chen LJ, Fox KR, Haase AM, Ku PW. Correlates of body dissatisfaction among Taiwanese adolescents. Asia Pac J Clin Nutr. 2010;19(2):172-9.

27- McCabe MP, Ricciardelli LA. Parent, peer, and media influences on body image and strategies to both increase and decrease body size among adolescent boys and girls. Adolescence. 2001;36(142):225-40.

28- Packard P, Krogstrand KS. Half of rural girls aged 8 to 17 years report weight concerns and dietary changes, with both more prevalent with increased age. J Am Diet Assoc. 2002;102(5):672-7.

29- Xu X, Mellor D, Kiehne M, Ricciardelli LA, McCabe MP, $\mathrm{Xu}$ Y. Body dissatisfaction, engagement in body change behaviors and sociocultural influences on body image among Chinese adolescents. Body Image. 2010;7(2):15664.

30- Dehghani M, Chehrzad MM, Jafari Asl M. Investigating the relationship between satisfaction from body image and socio cultural patterns among female adolescents in Rasht city. Sci J Hamadan Nurs Midwifery Fac. 2012;20(3):26-36. [Persian]

31- Kornblau IS, Pearson HC, Breitkopf CR. Demographic, behavioral, and physical correlates of body esteem among low-income female adolescents. J Adolesc Health. 2007;41(6):566-70.

32- von Rauste WM. Body image satisfaction in adolescent girls and boys: A longitudinal study. J Youth Adolesc. 1988;18(1):71-83.

33- Pullen L, Modrcin MA, McGuire SL, Lane K, Kearnely $\mathrm{M}$, Engle S. Anger in adolescent communities: How angry are they? Pediatr Nurs. 2015;41(3):135-40.

34- Hardy SA, Walker LJ, Olsen JA, Woodbury RD, Hickman JR. Moral identity as moral ideal self: Links to adolescent outcomes. Dev Psychol. 2014;50(1):45-57.

35- Shukla A, Dixit T. Interpersonal communication among adolescents. J Psychosoc Res. 2015;10(2):32736.

36- Tatnell R, Kelada L, Hasking P, Martin G. Longitudinal analysis of adolescent NSSI: The role of intrapersonal and interpersonal factors. J Abnorm Child Psychol. 2014;42(6):885-96.

37- Kearney J, Bussey K. The longitudinal influence of self-efficacy, communication, and parenting on

دوره سץ، شماره ا، زمستان موس| daughters in guidance schools of Urmia. J Urmia Nurs Midwifery Fac. 2011;9(6):417-23. [Persian]

4- Moodi M, Zamanipour N, Sharifirad GR, Shahnazi H. Evaluating puberty health program effect on knowledge increase among female intermediate and high school students in Birjand, Iran. J Educ Health Promot. 2013;2:57. [Persian]

5- Ybarra ML, Emenyonu N, Nansera D, Kiwanuka J, Bangsberg DR. Health information seeking among Mbararan adolescents: results from the Uganda Media and You survey. Health Educ Res. 2008;23(2):249-58.

6- Turkashvand R, Kermanshahi S, Azadfalah P. The effect of educational group therapy plan on self-esteem rate in adolescent girls. Zahedan J Res Med Sci. 2011;13(3):30-4. [Persian]

7- Weiler RM. Adolescents' perceptions of health concerns: An exploratory study among rural midwestern youth. Health Educ Behav. 1997;24(3):287-99.

8- Kodositabar M, Sadri M, Zanily Z, Ibrahim Z, Sahrawi E. Relationship between motor proficiency and selfconcept in girl students 11-14 ages. Annal Biol Res. 2012;4(6):276-80.

9- Heidari Soureshjani S, Eslam A, Alidousti M, Khosravi F, Hashemi F. Comparison the self-concept and behavior in adolescents without criminal record with juvenile offenders. J Shahrekord Univ Med Sci. 2014;15(6):32-40. [Persian]

10- Claes L, Houben A, Vandereycken W, Bijttebier P, Muehlenkamp J. Brief report: The association between non-suicidal self-injury, self-concept and acquaintance with self-injurious peers in a sample of adolescents. J Adolesc. 2010;33(5):775-8.

11- Karahroudy Alaee F, Kiani M, Izadi A, Majd Alavi H. Self-concept in normal and criminal male adolescents: A comparative study. J Shahid Beheshti Sch Nurs Midwifery. 2010;20(69):10-5. [Persian]

12- Corte C, Zucker RA. Self-concept disturbances: Cognitive vulnerability for early drinking and early drunkenness in adolescents at high risk for alcohol problems. Addict Behav. 2008;33(10):1282-90.

13- Froming WJ, Nasby W, McManus J. Prosocial selfschemas, self-awareness, and children's prosocial behavior. J Pers Soc Psychol. 1998;75(3):766-77.

14- Anoosheh M, Niknami S, Tavakoli R, Faghihzadeh S. Preliminary study of puberty education in adolescent girls: A qualitative research. Iran J Psychiatry Clin Psychol. 2003;9(2):64-70. [Persian]

15- Nik Azin A, Shaeeri MR, Nainian MR. Health related quality of life in adolescents: Mental health, socioeconomic status, gender, and age differences. J Iran Psychol. 2013;9(35):271-81. [Persian]

16- Cavallo F, Zambon A, Borraccino A, Raven-Sieberer U, Torsheim T, Lemma P. Girls growing through adolescence have a higher risk of poor health. Qual Life Res. 2006;15(10):1577-85.

17- Sadeghian E, Moghadari KM, Gorji S. The study of mental health status in high school female students in Hamadan city. Sci J Hamadan Univ Med Sci. 2010;17(3):39-45. [Persian]

18- Shakibaei Z, Tahmasebi R, Noroozi A. Determinant factors of mental health based on social cognitive theory among high school girl students of Bushehr. Iran J Health Educ Health Promot. 2014;2(2):131-42. [Persian]

19- Zareipour MA, Eftekhar AH, Azam K, Movahed E. Study of mental health and its relationship with family welfare in pre-university students in Salmas city in 2010. J Res Dev Nurs Midwifery. 2012;9(1):84-93. [Persian] 


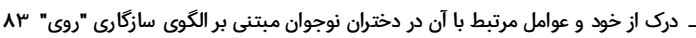

39- Hosseini M, Bayat R, Yaghmaei F, Hosseinzadeh S,

Nasiri N. The correlation of family functioning

spontaneous adolescent disclosure. J Res Adolesc. dimensions and self-concept of adolescent smokers in

Zanjan. J Health Promot Manag. 2012;1(4):61-8.

[Persian]

2015;25(3):506-23.

38- Ybrandt $\mathrm{H}$. The relation between self-concept and social functioning in adolescence. $J$ Adolesc. 2008;31(1):1-6. 